\title{
Interprofessional education for internationally educated health professionals: an environmental
}

\section{scan}

\author{
Mubashir Arain' \\ Esther Suter' \\ Sara Mallinson' \\ Shelanne L Hepp' \\ Siegrid Deutschlander ${ }^{\prime}$ \\ Shyama Dilani Nanayakkara ${ }^{2}$ \\ Elizabeth Louise Harrison ${ }^{3}$ \\ Grace Mickelson ${ }^{4}$ \\ Lesley Bainbridge ${ }^{5}$ \\ Ruby E Grymonpre 2 \\ 'Workforce Research \& Evaluation, \\ Alberta Health Services, Edmonton, \\ $\mathrm{AB},{ }^{2}$ College of Pharmacy, University \\ of Manitoba, Winnipeg, MB, ${ }^{3}$ School \\ of Physical Therapy, College of \\ Medicine, University of Saskatchewan, \\ Saskatoon, SK, ${ }^{4}$ Provincial Health \\ Services Authority, Vancouver, BC, \\ ${ }^{5}$ Department of Physical Therapy, \\ Faculty of Medicine, University of \\ British Columbia, Vancouver, BC, \\ Canada
}

This article was published in the following Dove Press journal:

Journal of Multidisciplinary Healthcare

3 March 2017

Number of times this article has been viewed

Objective: The objective of this environmental scan was to identify Western Canadian interprofessional education (IPE) resources that currently exist for internationally educated health professionals (IEHPs).

Methodology: A web-based search was conducted to identify learning resources meeting defined inclusion criteria with a particular focus on the resources available in the Western Canadian provinces. Information was extracted using a standardized template, and we contacted IEHP programs for additional information if necessary. Members of the research team reviewed preliminary findings, identified missing information from their respective provinces, and contacted organizations to fill in any gaps.

Results: The scan identified 26 learning resources for IEHPs in Western Canadian provinces and 15 in other provinces focused on support for IEHPs to meet their profession-specific licensing requirements and to acquire knowledge and competencies relevant to working in the Canadian health care system. Most learning resources, such as those found in bridging programs for IEHPs, included an orientation to the Canadian health care system, components of cultural competence, and at least one aspect of interprofessional competence (eg, communication skills). None of the 41 learning resources provided comprehensive training for IEHPs to cover the six interprofessional competency domains defined in the Canadian Interprofessional Health Collaborative (CIHC) National Interprofessional Competency Framework.

Conclusion: The IEHPs learning resources in Western Canada do not cover all of the interprofessional competencies. This review points to the value of developing a comprehensive IPE curriculum, based on the six domains identified in the CIHC National Interprofessional Competency Framework.

Keywords: health care personnel, human resource, collaborative practice, international medical graduates, Western Canadian provinces

\section{Introduction}

Interprofessional collaboration (IPC) is defined as the process through which different professional groups work together to positively impact health care. ${ }^{1}$ Working interprofessionally implies that health care professionals work more effectively to improve patient outcomes. ${ }^{2}$ With increasingly complex health care, IPC is essential to optimize the complementary skills of various health professionals. ${ }^{3}$ In 2010 , the World Health Organization (WHO) developed a framework for action on interprofessional education (IPE) and collaborative practice for decision makers, health providers, educators, and policy makers to embed it in all their services. ${ }^{3}$ IPE is the process by which we train practitioners to improve collaboration among health care providers. ${ }^{4}$ IPE helps with
Correspondence: Mubashir Arain Alberta Health Services, I030I Southport Tower, Calgary, AB T2W IS7, Canada Tel + I 4039430783

Email MubashirAslam.Arain@ahs.ca 
achieving IPC, which leads to improved patient-centered care and patient outcomes as well as better retention of health care providers. ${ }^{5-7}$ The WHO framework also proposed IPE and collaborative practice as important strategies to mitigate the shortage of health care workforce.

Canada has made significant efforts to advance IPE and IPC in the classroom and the workplace. The Medical Council recently released a blueprint that highlights collaboration and communication with other health care providers and the patients for shared care planning as an essential component of a physician's scope of practice. The blueprint states that physicians are expected to demonstrate these competencies and should be evaluated at entry into practice as well as at entry into independent practice. ${ }^{8}$ From 2005 to 2008 , Health Canada supported the Interprofessional Education for Collaborative Patient-Centred Practice (IECPCP) initiative. ${ }^{9}$ At the time of Health Canada's IECPCP initiative, participating academic institutions developed their own interprofessional competency frameworks, making comparisons of interprofessional competency acquisition across jurisdictions difficult. Under the auspices of the Canadian Interprofessional Health Collaborative (CIHC), a broad stakeholder group defined and described a common set of interprofessional competency domains. ${ }^{10}$ Many academic institutions and health service organizations have adopted these CIHC interprofessional competency domains to guide their work on advancing IPE and IPC. The six CIHC competency domains include the following: 1) interprofessional communication; 2) patient-/ client-centered and family-/community-centered care; 3) role clarification; 4) team functioning; 5) collaborative leadership; and 6) interprofessional conflict resolution.

In the past decade, nearly $25 \%(\sim 17,600)$ of Canadian physicians were born and trained outside Canada, and about $7 \%(360,000)$ of the Canadian nursing workforce had been trained abroad. ${ }^{11}$ Internationally educated health professionals (IEHPs) are working in almost every regulated and unregulated health profession in Canada. ${ }^{12}$ There are standards and licensing pathways which test the knowledge and competencies of IEHPs specific to the regulated professional requirements prior to entry into clinical practice. ${ }^{13,14}$ However, the prior education and practice of IEHPs vary significantly. ${ }^{15}$ Some health professionals come from very collaborative working environments, while other IEHPs might not have experienced the benefits of IPE and IPC. Therefore, helping IEHPs to acquire collaborative practice competencies is one important aspect in fostering effective collaboration among health care providers in Canada.
In 2013-2014, representatives from provincial health ministries in the four western provinces and northern territories identified a common interest in advancing the development of interprofessional competencies for IEHPs in their jurisdictions. Due to funding, timelines, and the education, practice, and regulatory partners, the scope of the detailed examination of the findings from the scan focused on Western Canadian resources. The purpose of this project was to develop an IPE curriculum for IEHPs in Western Canada. As a first step to the project, we conducted an environmental scan to understand the range of existing IPE learning resources specifically designed for IEHPs in Western Canadian provinces (Alberta, British Columbia, Saskatchewan, and Manitoba). The objective of the environmental scan was to examine the key components of learning resources for IEHPs and identify potential gaps.

\section{Methodology}

We conducted an environmental scan to identify the gaps in current IPE provided to IEHPs. Environmental scans allow the researcher, clinician, or policy maker to account for diverse types of knowledge. Environmental scans are now a recognized and valuable tool that provides evidence for policymaking, and program planning. ${ }^{16} \mathrm{~A}$ web-based search was conducted from January 2015 to March 2015 to identify learning resources for IEHPs on the Canadian health care system in Western Canadian provinces. We also included nationally available resources to position this work within the larger Canadian context. The search focused on seven regulated health professions: family physicians, licensed practical nurses (LPNs), registered nurses (RNs), registered psychiatric nurses (RPNs), occupational therapists, pharmacists, and physical therapists. These seven professions were chosen based on a number of factors. First, the government organizations from the four western provinces and northern territories identified these seven groups as representing larger numbers of IEHPs in their settings. Second, the project also involved a robust knowledge translation component; the team engaged and communicated with stakeholders involved in regulation and education of these health professions in each jurisdiction. In order to have meaningful connections with the range of stakeholders and considering the time and funding available to communicate and seek feedback, we focused on these seven groups.

We defined learning resource as any material (eg, bridging programs, modules, curricula) that had content aimed at improving IPC knowledge and competencies of IEHPs. Search terms included internationally educated health 
professionals, international medical graduates, family physicians, internationally educated pharmacists, internationally educated nurses, internationally educated physical therapists, internationally educated physiotherapist, internationally educated social workers, internationally educated occupational therapists, interprofessional education, interprofessional collaboration, communication skills for health professionals, bridging programs for health professionals in Canada, and orientation programs for health professionals. We excluded profession-specific clinical programs focused on teaching profession-specific clinical skills.

We identified the information about learning resources through these various sources: host websites using Google as a primary search engine, and MEDLINE/PubMed to identify published IEHP curricula. The research team members (MA, SM, SH, SD) also contacted Canadian universities, community colleges, and national and provincial health professional societies to retrieve any relevant curriculum on IPE for IEHPs. The information about learning resources was summarized into an extraction template that included the following variables: target population, types of competencies covered in the learning resource (eg, cultural competency, interprofessional competencies, and understanding of the Canadian health care system), delivery format (online/face-to-face), and program/course/module length. As the scan relied mainly on web-based information, all research team members (from all four Western Canadian provinces) reviewed the initial table to validate information based on their personal knowledge and to highlight missing information in their jurisdictions. We also engaged regulatory bodies in the four western provinces for the seven health professions throughout the study to identify any relevant information.

\section{Results}

The scan identified 26 learning resources for IEHPs in the Western Canadian provinces: seven in Alberta, five in British Columbia, six in Saskatchewan, and eight in Manitoba (Table 1). Fifteen additional learning resources were identified in other provinces and territories. Most of these Western Canadian as well as national resources were developed for internationally trained nurses and medical graduates. The content of these learning resources focused on orientation to Canadian health care system, cultural aspects of care, or on certain aspects of interprofessional practice/education, most commonly communication (Figure 1). None of the learning resources covered all six competency domains outlined in the CIHC National Competency Framework. Eighteen learning resources also included an orientation to the Canadian health care system (Figure 1). Supplementary material provides details of each learning resource.

\section{Courses for IEHPs - focus, teaching style, and length}

The scan identified that more learning resources were available for internationally educated nurses than any other professionals. Learning resources for internationally educated nurses were not specific to the three streams of nursing in Canada: RN, RPN, and LPN. Further, some of the resources contained general content such as working in a team, addressing conflicts among team members, which was relevant to any Canadian health care professional. While we identified resources with credit unit courses leading to certificates or diplomas, there were also some noncredit courses on specific topics such as communication skills. Most IEHP learning resources were face-to-face $(65 \% ; n=27)$, some used a blended (face-to-face and online) learning approach $(20 \% ; n=8)$, and a smaller number used exclusively online

Table I Number of learning resources offered in Western Canadian provinces and other provinces for IEHPs (N=4I)

\begin{tabular}{|c|c|c|c|c|c|}
\hline Professional groups & Alberta & British Columbia & Saskatchewan & Manitoba & Other provinces \\
\hline RN & 1 & I & I & 3 & 5 \\
\hline LPN & 2 & I & I & I & 0 \\
\hline RPN & 0 & I & 0 & 0 & 1 \\
\hline IMG & 1 & I & 3 & 1 & 3 \\
\hline OT & 0 & 0 & $\mathrm{I}^{\mathrm{a}}$ & 0 & $2^{\mathrm{b}}$ \\
\hline PT & I & 0 & 0 & 0 & 1 \\
\hline Pharmacy & I & I & 0 & I & 0 \\
\hline $\begin{array}{l}\text { Content not specific } \\
\text { to any profession }\end{array}$ & I & 0 & 0 & 2 & 4 \\
\hline Total & 7 & 5 & 6 & 8 & 15 \\
\hline
\end{tabular}

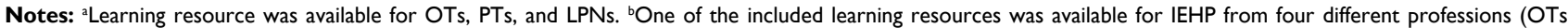
dieticians, RNs, PTs, and social workers).

Abbreviations: IEHP, internationally educated health professional; RN, registered nurse; LPN, licensed practical nurse; RPN, registered psychiatric nurse; IMG, internationally educated medical graduate; OT, occupational therapist; PT, physical therapist. 


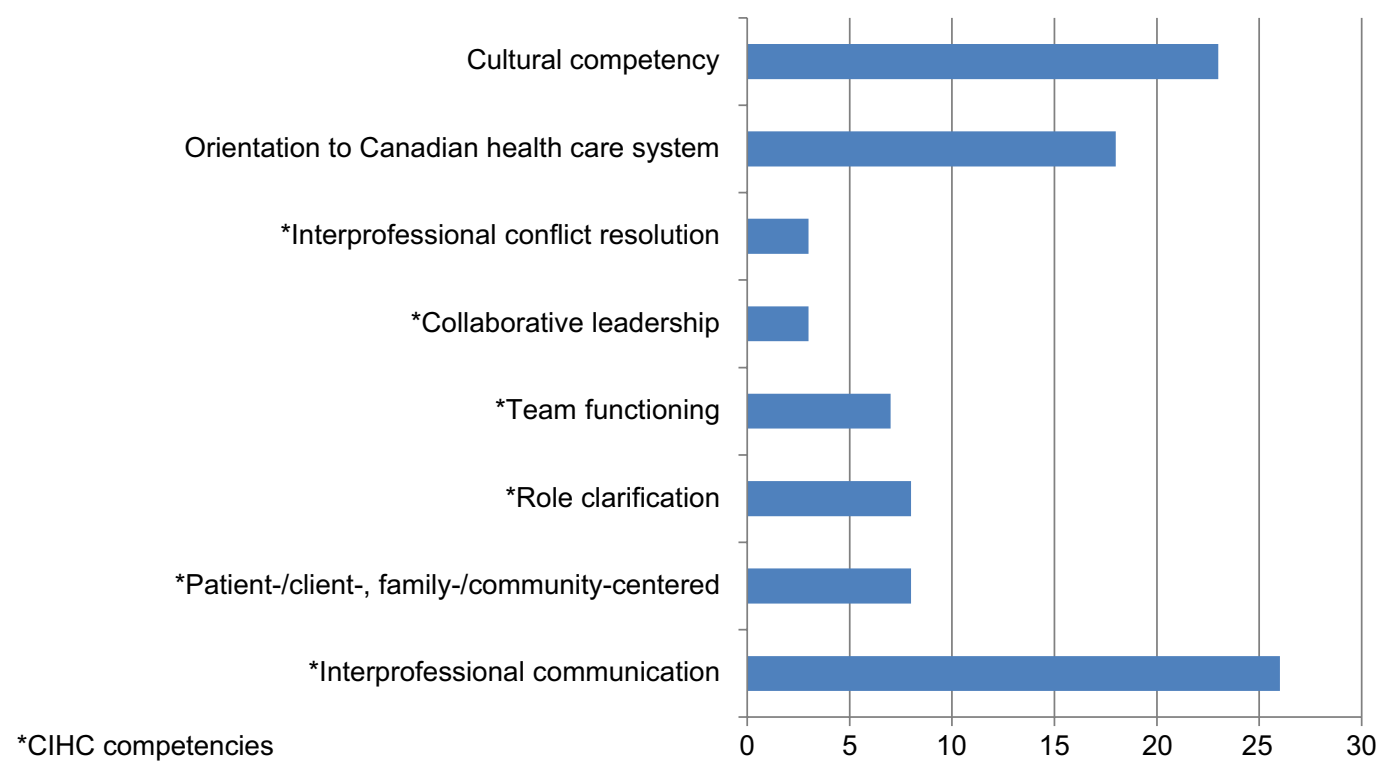

Figure I Mapping frequencies of different IP components covered in the learning resources for IEHPs (N=4I).

Abbreviations: IP, interprofessional; IEHPs, internationally educated health professionals; CIHC, Canadian Interprofessional Health Collaborative.

courses/modules delivery $(15 \% ; n=6)$. Credit courses were offered for anywhere between seven and 36 months, while noncredit courses tended to last fewer than 12 weeks. Some interprofessional learning opportunities for IEHPs included seminars, conferences, and mentorship in clinical practice environments. Some learning resources were shared between different postsecondary institutions.

\section{Interprofessional competency domains covered}

It was not always possible to assess the extent of the interprofessional content from learning resource information available online. However, the scan showed a similar picture of learning resources in Western Canada and other provinces with interprofessional knowledge and skills being one of several components taught (Box 1). Most learning resources for IEHPs covered aspects of cultural diversity, interprofessional teamwork and collaborative practice (eg, communication skills between providers), and/or an introduction to Canadian health care (eg, history, culture) together with profession-specific content. Some academic learning resources for IEHPs including programs offered through NorQuest College, University of Alberta, Thompson Rivers University, University of British Columbia, University of Manitoba, Saskatchewan Polytechnic, and University of Saskatchewan explicitly referred to work on interprofessional teams. Cultural aspects of care were included in the learning resources offered at Mount Royal University, MacEwan University, NorQuest College, University of Alberta, Langara College, Kwantlen Polytechnic, Thompson Rivers University,
University of Manitoba, University of Saskatchewan, University of British Columbia, and Saskatchewan Polytechnic.

\section{Discussion}

This scan revealed a number of important characteristics of learning resources for IEHPs to foster their transition to IPC within the Canadian health care system. Most IEHP learning resources addressed cultural aspects of care (eg, cultural awareness and sensitivity about Canadian health care) and knowledge of the Canadian health care system. Bridging programs at community colleges do not have an explicit focus on IPC in their curriculum. A few learning resources covered some of the interprofessional competency domains. However, none of the learning resources covered all six collaborative practice competency domains as outlined in the CIHC National Interprofessional Competency Framework. These learning resources may have covered some content that could be linked to a domain, but it was not always clear as to whether it was in the context of IPC. The development of an IPE curriculum for IEHPs with learning objectives specific to development of IPC competencies and guided by the CIHC National Interprofessional Competency Framework would address this education gap.

In the current health care environment, competency acquisition that goes beyond learning profession-specific clinical skills has become an important priority in health professional education. ${ }^{17,18}$ Training in IPC is a required educational requirement for many Canadian-trained health professionals and reinforced through emerging accreditation standards for IPE in colleges and universities offering health professional 
Box I A brief summary of IEHP learning resources in Canadian context for IEHPs

\begin{abstract}
Alberta
Cultural diversity: One professional communication course for IEHPs offered by Mount Royal University and MacEwan University covered communication with culturally diverse patients and coworkers. The online University of Alberta course for physiotherapists covered cultural competence and interprofessional practice. The NorQuest diploma for internationally educated nurses (not currently running) had courses on intercultural communication and social determinants of health. Other courses include general introductions to communication and culture for nursing or professional communication skills.
\end{abstract}

Canadian health system: Most courses had an orientation to Canadian health care that covers history, culture, and the context of professional practice. Courses at Mount Royal, MacEwan, and NorQuest included a social science introduction to social determinants of health and community. Interprofessional competencies: While most courses did not specifically refer to interprofessional competencies or any national frameworks for IPE, they included communication skills. The diploma course for LPNs at NorQuest and the online course for PTs at University of Alberta referred to skills or competencies for working in interprofessional teams.

\title{
British Columbia
}

Cultural diversity: Kwantlen Polytechnic University had two courses that address personal beliefs, values, expectations, and cultural influences in professional nursing practice. Langara College also had a course on culture and health and how this influences nurse-client relationships. Kwantlen and Langara make explicit reference to aboriginal or indigenous people in these course outlines. Thompson Rivers included a course on diversity in families and communities. The University of British Columbia Canadian Pharmacy Practice Program included a course on patient dialog skills, which covered cultural diversity and communications and a session exploring the impact of diverse culture in the workplace.

Canadian health system: All courses include an orientation to Canadian health care. Langara College includes learning on social determinants of health and Canadian populations as well as Canadian nursing competencies and standards.

Interprofessional competencies: Key interprofessional skills such as effective communication and team-based care are included in some of the IEHP courses, but not all. Courses notable for their focus on working in teams (including effective working relationships, communication skills, and collaboration) are taught by Kwantlen Polytechnic University, Langara College, Thompson Rivers University, and University of British Columbia Canadian Pharmacy Practice Program. Other courses do not explicitly include topics related to IPE in program outlines.

\section{Manitoba}

Cultural diversity: Many courses do not include a specific module or learning goal on culture or cultural diversity. One exception is the University of Manitoba's IMG Program Orientation that provides sociocultural training.

Canadian health system: Courses that comprise an orientation to health care practice in Canada cover the organization of health care provision and professional roles and responsibilities.

Interprofessional competencies: The University of Manitoba's program for IMGs provides training on team-based practice. Other courses tend to focus on general communication skills.

\section{Saskatchewan}

Cultural diversity: Not all courses have an explicit focus on diversity or culture. The RN orientation course at Saskatchewan Polytechnic includes an exploration of issues arising from language barriers and cultural differences. The Polytechnic's module on health professional roles and ethics (offered as part of Saskatchewan Association of Licensed Practical Nurses registration preparation program) also covers content related to cultural diversity.

Canadian health system: Courses at Saskatchewan Polytechnic provide an orientation to professional practice in Canadian health care. A module offered by the Saskatchewan Pathways program also focuses on the Canadian workplace, as does the SIPPA program for IMGs at the University of Saskatchewan.

Interprofessional competencies: Most IEHP courses cover communication skills, although some focus on English language skill development for IEHPs. Some courses have a more explicit focus on team-based communications, most notably the Orientation to Nursing course at Saskatchewan Polytechnic, the online course on roles, responsibilities, and ethics at Saskatchewan Polytechnic, and the SIPPA program for IMGs at the University of Saskatchewan. The SIPPA program also includes teaching on interprofessional care and professional boundaries.

\section{Other provinces}

Cultural diversity: Courses for IEHPs on cultural aspect of care, cultural diversity, and its impact on professional practice are offered by the University of Toronto (courses for PTs and pharmacists) and Mohawk College (via McMaster University campus). The LPN bridging program at Conestoga College also teaches students about intercultural health issues, as does the medical license-bridging program offered in Ottawa in partnership with the University of Ottawa medical school (program under revision). The Registered Nurses Professional Development Centre in Halifax also covers culture and diversity in orientation for IEHP.

Canadian health system: Most of the courses and institutions named above include sessions on working in the Canadian health care system. Interprofessional competencies: The IPE for internationally educated nurses' toolkit (George Brown College) covers learning about the competencies for collaborative practice and care. It also offers guidance on leading and coordinating IPE for internationally educated nurses. The Orientation to Canadian Health Care System offered by the Registered Nurses Professional Development Centre also includes interprofessional practice. The Workplace Communication Skills for Interprofessional Health Care course at Conestoga College, Centennial College, and George Brown College includes communication for interprofessional health care teams. Moreover, Conestoga College's Bridge to Practical Nursing Course covers intra- and interprofessional practice in multidisciplinary teams as part of the module on professional nursing practice.

Abbreviations: IEHP, internationally educated health professional; IPE, interprofessional education; LPN, licensed practical nurse; PT, physical therapist; IMG, internationally educated medical graduate; RN, registered nurse; SIPPA, Saskatchewan International Physician Practice Assessment. 
programs. Various policies and guidelines have highlighted the integration of all sectors (eg, regulators, academics, policy makers, decision makers) to develop a common and meaningful agenda on IPC. ${ }^{10}$ IPE is as important for IEHPs as it is for Canadian health professionals. However, IPE for IEHPs must also be designed considering specific learner needs. The literature emphasizes that communication skills, cultural challenges, and the need for individual support are the key challenges of international graduates in a Canadian health care environment. One study on internationally educated medical graduates (IMGs) reported the need for educational courses to address communication skills and training issues related to the health care system. ${ }^{19}$ Another study mentioned that patient communication and communication with team members were the two biggest challenges for IMGs. ${ }^{14}$ Further, IEHPs face several additional problems that are less common to their Canadian counterparts. They often face social and financial issues, worries about visas/immigration, and concerns related to family members left behind in home countries. ${ }^{20}$ For many IEHPs, the cultural differences between their home country and Canada are significant, which may be a further barrier to effective IPC. ${ }^{21}$ Cultural competence has been defined as "the ability of individuals to establish effective interpersonal and working relationships that supersede cultural differences". ${ }^{22}$ Cultural competency of health professionals supports better IPC environments in health care systems. ${ }^{23}$

Providing IPE to IEHPs may also assist with improving the understanding of the Canadian health care system as context (eg, practice settings, patient populations) is frequently referenced in IPC educational programming. The content of interprofessional courses should cover all competency domains according to Health Canada and the CIHC National Interprofessional Competency Framework to meet the needs of IEHPs. Although existing interprofessional learning resources can serve as a key reference, additional content particularly focusing on cultural competency along with the six IP competencies is required to meet the unique requirements of IEHPs. Exemplary international resources can certainly help in developing the content; ${ }^{24,25}$ the Health Workforce Australia has recently produced a report, National Common Health Competency Resource for the Australian Health Workforce, which specifies competencies believed to be common between health professions "as a tool for employers to inform and support flexible approaches to workforce design and redesign". ${ }^{26}$

We identified some limitations of this environmental scan. First, the scan was only focused on the Western Canadian jurisdictions; some other common resources were accessed to position this work within the broader Canadian context, but the scan was mainly limited to Western Canada. Second, some learning resources did not have enough information online about the content. We contacted relevant institutes to obtain additional information where possible. Third, the scan was limited to seven health professions. However, most learning resources included in this paper are nonspecific to any profession, and the information can be used by other health professionals as well. Despite these limitations, the scan provided very useful information about the learning resources available for IEHPs in Canada.

Our scan identified the need for a comprehensive IPE curriculum for IEHPs in Western Canada. The curriculum should be focused on IPC including all six competency domains of the CIHC National Interprofessional Competency Framework along with cultural competency. The curriculum would provide foundational knowledge in interprofessional collaborative practice with the ultimate goal of more efficient integration of IEHPs into the Canadian health care system.

\section{Conclusion}

Our scan identified a number of learning resources for IEHPs in Canada. Most learning resources include an orientation to the Canadian health care system, components of cultural competence, and one or more aspects of interprofessional competence. None of the learning resources provided comprehensive training for IEHPs to cover all six interprofessional competency domains. This review points to the value of developing a comprehensive IPE curriculum, based on the six domains identified in the CIHC National Interprofessional Competency Framework.

\section{Acknowledgments}

This project has been undertaken with the support and collaboration of the Western and Northern Health Human Resources Planning Forum and its member jurisdictions, and has been made possible through a financial contribution from Health Canada. The views expressed herein do not necessarily represent the views of Health Canada.

\section{Disclosure}

The authors report no conflicts of interest in this work.

\section{References}

1. Zwarenstein M, Goldman J, Reeves S. Interprofessional collaboration: effects of practice-based interventions on professional practice and healthcare outcomes. Cochrane Database Syst Rev. 2009;(3):CD000072. 
2. Reeves S, Goldman J, Gilbert J, et al. A scoping review to improve conceptual clarity of interprofessional interventions. J Interprof Care. 2011;25(3):167-174.

3. The World Health Organization. Framework for action on interprofessional education \& collaborative practice. 2010. Available from: http:// www.who.int/hrh/resources/framework_action/en/. Accessed October 20,2016

4. Bainbridge L, Wood VI. The power of prepositions: learning with, from and about others in the context of interprofessional education. $J$ Interprof Care. 2012;26(6):452-458.

5. Wilbur K, Kelly I. Interprofessional impressions among nursing and pharmacy students: a qualitative study to inform interprofessional education initiatives. BMC Med Educ. 2015;15:53.

6. Reeves S, Perrier L, Goldman J, Freeth D, Zwarenstein M. Interprofessional education: effects on professional practice and healthcare outcomes (update). Cochrane Database Syst Rev. 2013;(3):CD002213.

7. Health Canada. Program evaluation for interprofessional initiatives: evaluation instruments/methods of the 20 IECPCP projects. 2009 Available from: http://www.cihc.ca/files/CIHC_EvalMethods_Final. pdf. Accessed October 20, 2016.

8. Touchie C, Streefkerk C, for the Blueprint Project Team. Blueprint Project - Qualifying Examinations Blueprint and Content Specifications. Ottawa, Ontario. September 2014. Available from: http://mcc ca/wp-content/uploads/Blueprint-Report.pdf. Accessed December 12, 2016.

9. Silver IL, Leslie K. Faculty development for continuing interprofessional education and collaborative practice. J Contin Educ Health Prof. 2009;29(3):172-177.

10. A National Interprofessional Competency Framework. Canadian Interprofessional Health Collaborative. Health Canada. 2010. Available from: http://www.cihc.ca/files/CIHC_IPCompetencies_Feb1210.pdf. Accessed October 24, 2016.

11. Neiterman E, Bourgeault IL. Professional integration as a process of professional resocialization: internationally educated health professionals in Canada. Soc Sci Med. 2015;131:74-81.

12. Health Canada. Pan-Canadian Health Human Resource Strategy 2007-2008 annual report. Available from: http://www.hc-sc.gc.ca/hcssss/pubs/hhrhs/2008-ar-ra/index-eng.php. Accessed October 20, 2016.

13. Greig A, Dawes D, Murphy S, Parker G, Loveridge B. Program evaluation of a model to integrate internationally educated health professionals into clinical practice. BMC Med Educ. 2013;13:140.
14. Mulholland SJ, Dietrich TA, Bressler SI, Corbett KG. Exploring the integration of internationally educated occupational therapists into the workforce. Can J Occup Ther. 2013;80(1):8-18.

15. Zulla R, Baerlocher MO, Verma S. International medical graduates (IMGs) needs assessment study: comparison between current IMG trainees and program directors. BMC Med Educ. 2008;8:42.

16. Graham P, Evitts T, Thomas-MacLean R. Environmental scans: how useful are they for primary care research? Can Fam Physician. 2008;54(7):1022-1023.

17. Owen JA, Schmitt MH. Integrating interprofessional education into continuing education: a planning process for continuing interprofessional education programs. J Contin Educ Health Prof. 2013;33(2):109-117.

18. Pecukonis E, Doyle O, Bliss DL. Reducing barriers to interprofessional training: promoting interprofessional cultural competence. J Interprof Care. 2008;22(4):417-428.

19. Hall P, Keely E, Dojeiji S, Byszewski A, Marks M. Communication skills, cultural challenges, and individual support: challenges of international medical graduates in Canadian healthcare environment. Med Teach. 2004;26(2):120-125

20. Majumdar B, Keystone JS, Cuttress LA. Cultural sensitivity training among foreign medical graduates. Med Educ. 1999;33(3):177-184.

21. Sockalingam S, Khan A, Tan A, et al. A framework for understanding international medical graduate challenges during transition into fellowship programs. Teach Learn Med. 2014;26(4):401-408.

22. Cooper LA, Roter DL. Patient-provider communication: the effect of race and ethnicity on process and outcomes of healthcare. In: Smedley BD, Stith AY, Nelson AR, editors. Unequal Treatment: Confronting Racial and Ethnic Disparities in Healthcare. Washington, DC: The National Academies Press; 2002: 552-593.

23. Purden M. Cultural considerations in interprofessional education and practice. J Interprof Care. 2005;19(Suppl 1):224-234.

24. Thistlethwaite JE, Forman D, Matthews LR, Rogers GD, Steketee C, Yassine T. Competencies and frameworks in interprofessional education: a comparative analysis. Acad Med. 2014;89(6):869-875.

25. Steketee C, Forman D, Dunston R, et al. Interprofessional health education in Australia: three research projects informing curriculum renewal and development. Appl Nurs Res. 2014;27(2):115-120.

26. Health Workforce Australia. National Common Health Competency Resource for the Australian Health Workforce. 2013. Available from http://www.tcen.com.au/sites/newtcen/files/files/CSSP/nationalcommon-health-capability-resource.pdf. Accessed December 12, 2016.
Journal of Multidisciplinary Healthcare

\section{Publish your work in this journal}

The Journal of Multidisciplinary Healthcare is an international, peerreviewed open-access journal that aims to represent and publish research in healthcare areas delivered by practitioners of different disciplines. This includes studies and reviews conducted by multidisciplinary teams as well as research which evaluates the results or conduct of such teams or health
Dovepress

care processes in general. The journal covers a very wide range of areas and welcomes submissions from practitioners at all levels, from all over the world The manuscript management system is completely online and includes a very quick and fair peer-review system. Visit http://www.dovepress.com/ testimonials.php to read real quotes from published authors. 